\title{
DEVELOPMENT AND EVALUATION OF MUCOADHESIVE MICROSPHERES OF LEVOFLOXACIN HYDROCHLORIDE
}

\author{
*Chaudhary Amit, Nagaich Upendra, Rastogi Bhavya, Gulati Neha \\ Department of Pharmaceutics, School of Pharmacy, Bharat Institute of Technology, Partapur, Meerut, Uttar Pradesh, India- 250103 \\ Corresponding Author's Email: amitub@rediffmail.com
}

Received 18 Sep 2012; Review Completed 24 Oct 2012; Accepted 06 Nov 2012, Available online 15 Nov 2012

\begin{abstract}
The aim of the present work is to prepare Levofloxacin microspheres with mucoadhesive polymers like Sodium Alginate, Sodium Carboxymethyl Cellulose and Carbopol-940.Microspheres were prepared by w/o emulsification solvent evaporation method and evaluated. The resulting microspheres were small, discrete, spherical and free flowing. The microspheres showed significant mucoadhesive property in the in-vitro wash-off test. The drug release from the mucoadhesive microspheres followed the controlled release profile. and first order kinetics. Drug release was controlled by the diffusion mechanism. Stability studies were performed at three different temperatures for six weeks. All the formulation showed satisfactory stability profile.

Keywords:Mucoadhesive, Microspheres, Carbopol-940, SCMC, Levofloxacin
\end{abstract}

\section{INTRODUCTION}

Mucodhesion is a promising approach in the design of drug delivery systems to prolong the residence time of the dosage form the site of application or absorption and to facilitate intimate contact of the dosage form with the underlying absorption surface to improve and enhance the bioavailability of drugs ${ }^{1}$. Several studies reported mucodhesive drug delivery systems in the form of tablet, films, patches and gels for oral, buccal, nasal, occular and topical routes.

Levofloxacin hydrochloride is a fluoroquinolone antibacterial drug, which is used in the various kinds of infections like urinary tract infections, gonorrhoea vaginitis etc ${ }^{2}$. It has very bitter taste and is readily soluble in the gastric $\mathrm{pH}$ due to the formations of salt. The drug is also reported to have poor stability at elevated temperatures and exposure to sunlight, due to the ring opening and formation of ethylene diamine and formamide derivative ${ }^{3}$. To over come with these problems, mucoadhesive microspheres of Levofloxacin were formulated using Sodium Alginate, Sodium Carboxymethyl Cellulose and Carbopol-940 polymers. These mucoadhesive polymers in the microspheres prolong the residence time of the dosage form in the gastrointestinal tract and hence are more suitable as matrix material for oral controlled release ${ }^{4}$.

\section{MATERIALS AND METHODS}

Levofloxacin hydrochloride was a gift sample from Cipla Ltd. Mumbai. Sodium Carboxymethyl Cellulose (1500400cps) was procured from Central Drug House, Mumbai. Carbopol-940 and Sodium Alginte was procured from Loba Chemie, Mumbai. Light liquid paraffin and Span-20 were procured from commercial sources. All other reagents used were of analytical grade.

\section{Preparation of mucoadhesive microspheres:}

Microspheres were prepared by the water-in-oil (w/o) emulsification solvent evaporation technique. The model drug was dissolved in each polymeric aqueous solutions.
The solutions were poured into $200 \mathrm{~g}$ of mineral oil containing $0.5 \%$ span -20 as an emulsifying agent. The aqueous phase was emulsified into the oily phase by stirring the system in a $500 \mathrm{ml}$ beaker. Constant stirring at $2000 \mathrm{rpm}$ was carried out using mechanical stirrer (Remi, Model No.FTQ 124A). The beaker and its content were heated by an on the hot plate at $80{ }^{\circ} \mathrm{C}$. Stirring and heating were maintained for $4.5 \mathrm{~h}$ until the aqueous phase was completely removed by evaporation. The light mineral oil was decanted and collected microspheres were washed three times with $100 \mathrm{ml}$ aliquots of $\mathrm{n}$-hexane, filtered through Whatman filter paper, dried in an oven at $50{ }^{\circ} \mathrm{C}$ for $2 \mathrm{~h}$ and stored in a desiccator at room temperature. Microspheres were prepared using Sodium Alginate, Sodium Carboxymethyl Cellulose and Carbopol-940 polymers ${ }^{5}$. Compositions of formulation(s) are shown in Table 1.

\section{Drug content}

Levofloxacin content in the microspheres was estimated by a UV Spectrophotometric (Thermospectronic Model No.156) method based on the measurement of absorbance at $280 \mathrm{~nm}$ in distilled water. Microspheres equivalent to $50 \mathrm{mg}$ were weighed and added in $100 \mathrm{ml}$ of distilled water. The volumetric flask was stirred continuously for $24 \mathrm{~h}$ on a magnetic stirrer. Dilutions of above solutions were made suitably and measured for the drug content.

\section{Particle size:}

Particle size analysis was performed with the help of optical microscopy for all the formulations.

\section{In-Vitro Drug Release Study:}

Release of Levofloxacin HCI from the microspheres was studied in distilled water using USP XXIII Six station dissolution test apparatus (Thermospectronic Model No.156) with rotating basket at $100 \mathrm{rpm}$ and at $37^{\circ} \mathrm{C}$.

For each formulation, Microspheres equivalents to $100 \mathrm{mg}$ of Levofloxacin were filled in empty capsule shells. One 
Amit et al

Journal of Drug Delivery \& Therapeutics; 2012, 2(6), 21-24

capsule was used in each test and placed in the basket. Samples of dissolution fluid were withdrawn at different intervals, filtered and diluted suitably and then analyzed spectrophotometrically at $280 \mathrm{~nm}$.

(Fig.1, 2) show the graphical representation of in-vitro release profile from the formulations ${ }^{6}$.

\section{In-Vitro Test for Mucoadhesion:}

The mucoadhesion property of the microspheres was evaluated by a modified in-vitro wash off test method. Pieces of the intestinal mucosa $(3 \mathrm{~cm} \mathrm{x} 1 \mathrm{~cm})$ were mounted on to glass rods $(4 \mathrm{~cm} \mathrm{x} 1 \mathrm{~cm})$ and placed in the tubes of USP Tablet disintegrating test apparatus. 50 microspheres were spread on to each wet rinsed pieces of intestinal mucosa in distilled water ${ }^{7}$. The tissue specimen was given a slow regular up and down movement in the test fluid at $37{ }^{\circ} \mathrm{C}$, up to $3.5 \mathrm{~h}$, after each half a hour the machine was stopped and the number of microspheres adhering on to the tissue were counted by using convex hand lens.

\section{Stability Studies}

Microspheres formulations were stored at three different temperatures of refrigerated, at room temperature and at $45{ }^{0} \mathrm{C}$ for 42 days $^{8}$. After six week of the storage of the microspheres Levofloxacin was analyzed in consecutive order for the drug content spectrophotometrically at 280 nm. Data of stability studies are shown graphicaly at different temperatures respectively.

\section{RESULT AND DISCUSSION}

Emulsion Solvent Evaporation Method using Sodium Carbooxymethyl Cellulose, Sodium Alginate and Carbopol-940 prepared the Mucoadhesive microspheres of Levofloxacin hydrochloride. Microspheres were designed using different ratios of the polymers as shown in Table given below

Table 1: Composition of Mucoadhesive Microspheres

\begin{tabular}{|l|l|l|l|l|l|l|}
\hline Ingredient (g) & $\mathbf{F}_{\mathbf{1}}$ & $\mathbf{F}_{\mathbf{2}}$ & $\mathbf{F}_{\mathbf{3}}$ & $\mathbf{F}_{\mathbf{4}}$ & $\mathbf{F}_{\mathbf{5}}$ & $\mathbf{F}_{\mathbf{6}}$ \\
\hline Drug (LevofloxacinHCl) & 1 & 1 & 1 & 1 & 1 & 1 \\
\hline Carbopol-940 & - & 1 & - & 0.5 & - & 0.5 \\
\hline Sodium Carboxymethyl Cellulose & 1 & - & - & 0.5 & 0.5 & - \\
\hline Sodium Alginate & - & - & 1 & - & 0.5 & 0.5 \\
\hline
\end{tabular}

Table 2: Drug Content in different Formulations

\begin{tabular}{|c|c|c|c|}
\hline Formulations & Theoretical loading (mg) & Practical yield (mg) & \% Drug loading \\
\hline $\mathrm{F}_{1}$ & 50 & 32 & $64 \pm 1.50$ \\
\hline $\mathrm{F}_{2}$ & 50 & 38 & $76 \pm 2.50$ \\
\hline $\mathrm{F}_{3}$ & 50 & 26 & $52 \pm 3.40$ \\
\hline $\mathrm{F}_{4}$ & 50 & 37 & $74 \pm 2.40$ \\
\hline $\mathrm{F}_{5}$ & 50 & 29 & $58 \pm 2.95$ \\
\hline $\mathrm{F}_{6}$ & 50 & 25 & $50 \pm 4.5$ \\
\hline
\end{tabular}

Table 2 shows drug content in all different Formulations were estimated by UV Spectrophotometric method, Percent loading efficiency were found in the range of 50-76\%.

Formulation $\mathrm{F}_{2}$ containing carbopol-940 showed maximum percent loading of drug up to $76 \%$. Formulation $\mathrm{F}_{6}$ containing carbopol-940 / Sodium Alginate showed less percent loading of drug as compared to Formulation $\mathrm{F}_{2}$. Rank order of Percent loading was found to be as followed. $\mathrm{F}_{2}>\mathrm{F}_{4}>\mathrm{F}_{1}>\mathrm{F}_{5}>\mathrm{F}_{3}>\mathrm{F}_{6}$.

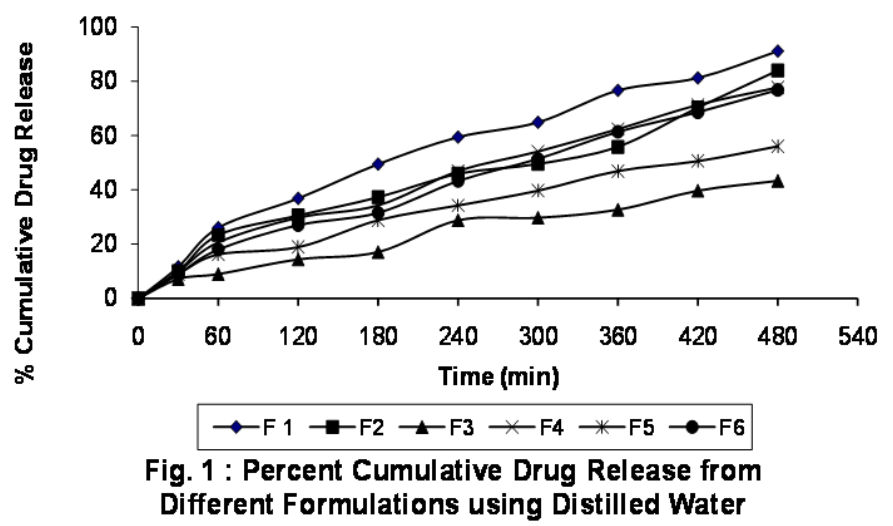

Particle size analysis of different formulations was done by optical microscopy. The average particle size was found to be in the range of $27.9 \mu \mathrm{m}-35.75 \mu \mathrm{m} . \mathrm{F}_{2}$ showed the least particle size of $27.9 \mu \mathrm{m}$ which is due to spherical nature of microspheres as showed by the SEM. Sodium alginate showed the largest particle size of $35.75 \mu \mathrm{m}$, due to the irregular nature of the microspheres, confirmed by SEM.
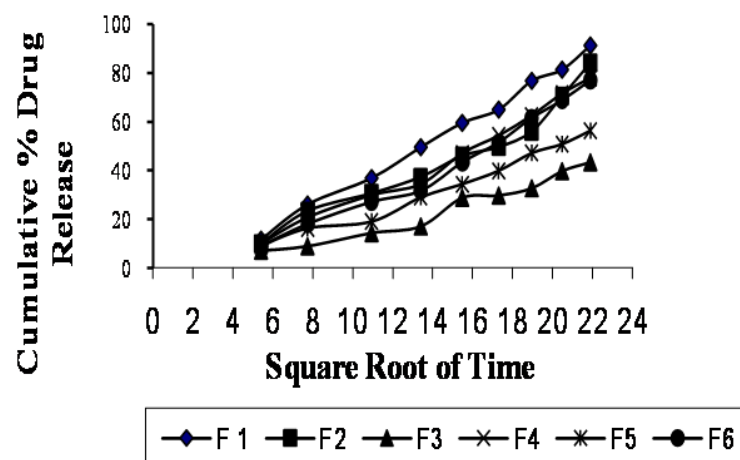

Fig. 2 : Cumulative Percent Drug Release Versus Square Root of Time of Different Formulations 
Amit et al

Journal of Drug Delivery \& Therapeutics; 2012, 2(6), 21-24

In-vitro release studies of all the formulations containing drug were performed in distilled water at $280 \mathrm{~nm}$. It was found that the drug release from the Formulations were distinguishably different for the different polymers used in the Formulations. After the end of $8 \mathrm{~h}$ the release were found to be 91.26, 84.14, 43.39, 77.86, 57.07 and 76.84 percent of formulations $\mathrm{F}_{1}, \mathrm{~F}_{2}, \mathrm{~F}_{3}, \mathrm{~F}_{4}, \mathrm{~F}_{5}$ and $\mathrm{F}_{6}$ respectively . Formulation $\mathrm{F}_{1}$ containing SCMC showed the maximum release after the $8 \mathrm{~h}$ and due to high swelling property of SCMC. While Sodium Alginate microspheres $\left(\mathrm{F}_{3}\right)$ showed less drug release pattern after $8 \mathrm{~h}$ due to less swelling action and irregular surface as compared to SCMC

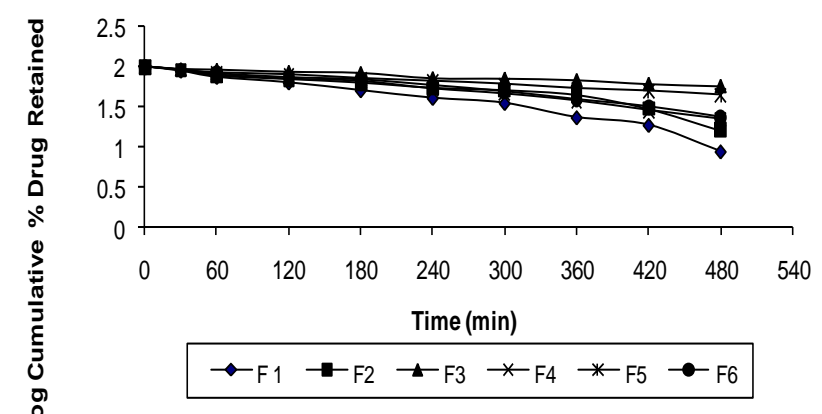

Fig. 3 : Log Cumulative Percent Drug Retained in Different Formulations
Higuchi plots were plotted for the formulations to determine the mechanism of drug release pattern fig 2 shows the graphical representation of cumulative percentage drug release versus square root of time for all the formulations. These Higuchi's Plots were found to be linear with correlation coefficient values of 0.9977 , $0.9724,0.9799,0.9914,0.9915,0.9882$ for formulation $F_{1}$, $\mathrm{F}_{2}, \mathrm{~F}_{3}, \mathrm{~F}_{4}, \mathrm{~F}_{5}$ and $\mathrm{F}_{6}$ respectively. The linearity indicated that the drug release from the microspheres followed the matrix diffusion process.

The plots of log cumulative percent drug retained versus time were found to be linear for all the formulations his linearity indicates that the release of Levofloxacin from the microspheres have obeyed first order kinetics films might have followed first order kinetics. The correlation coefficient values were found to be $-0.9770,-0.9454$, $0.9915,-0.9969,-0.9906,-0.9867$ for formulation $F_{1}, F_{2}$, $\mathrm{F}_{3}, \mathrm{~F}_{4}, \mathrm{~F}_{5}$ and $\mathrm{F}_{6}$ respectively. It shows that data are in good correlation. Negative values of the correlation coefficient indicate negative slope for the plot.

To assess the mucoadhesivity of the microspheres in-vitro wash off test was performed for all the formulations. At the end of $3.5 \mathrm{~h}$ Percent mucoadhesivity was found to be $30,16,4,10,10,4$ for formulation $F_{1}, F_{2}, F_{3}, F_{4}, F_{5}$ and $F_{6}$ respectively Table 3 .

Table 3: Results of In-Vitro Wash Off Test to Assess Mucoadhesive Property of the Microspheres

\begin{tabular}{|c|c|c|c|c|c|c|c|}
\hline \multirow[t]{2}{*}{ Formulations } & \multicolumn{7}{|c|}{ Percent of Microspheres Adhering to Tissue at 6 times(min) in Distilled Water } \\
\hline & 30 & 60 & 90 & 120 & 150 & 180 & 210 \\
\hline $\mathrm{F}_{1}$ & $86 \pm 2.54$ & $70 \pm 1.45$ & $62 \pm 0.97$ & $56 \pm 1.67$ & $40 \pm 1.35$ & $36 \pm 2.21$ & $30 \pm 0.67$ \\
\hline $\mathrm{F}_{2}$ & $84 \pm 0.98$ & $60 \pm 1.24$ & $40 \pm 1.67$ & $36 \pm 1.64$ & $26 \pm 0.99$ & $20 \pm 1.08$ & $16 \pm 1.90$ \\
\hline $\mathrm{F}_{3}$ & $80 \pm 1.45$ & $56 \pm 1.58$ & $49 \pm 0.64$ & $32 \pm 1.45$ & $24 \pm 2.02$ & $10 \pm 1.02$ & $4 \pm 0.57$ \\
\hline $\mathrm{F}_{4}$ & $64 \pm 0.97$ & $62 \pm 1.24$ & $46 \pm 2.05$ & $32 \pm 1.34$ & $24 \pm 0.97$ & $12 \pm 0.66$ & $10 \pm 1.02$ \\
\hline $\mathrm{F}_{5}$ & $90 \pm 2.25$ & $78 \pm 1.65$ & $64 \pm 0.99$ & $54 \pm 1.86$ & $20 \pm 1.05$ & $10 \pm 0.64$ & $10 \pm 0.63$ \\
\hline $\mathrm{F}_{6}$ & $6 \pm 1.23$ & $44 \pm 1.54$ & $40 \pm 1.02$ & $30 \pm 2.34$ & $20 \pm 2.04$ & $8 \pm 0.65$ & $4 \pm 1.02$ \\
\hline
\end{tabular}

Formulation $\mathrm{F}_{1}$ showed the highest percent mucoadhesion. The greater mucoadhesivity of the SCMC microspheres were due to anionic nature of the polymer, and sodium alginate showed the lowest percent mucoadhesion due to the irregular surface microspheres prepared by it. The rank

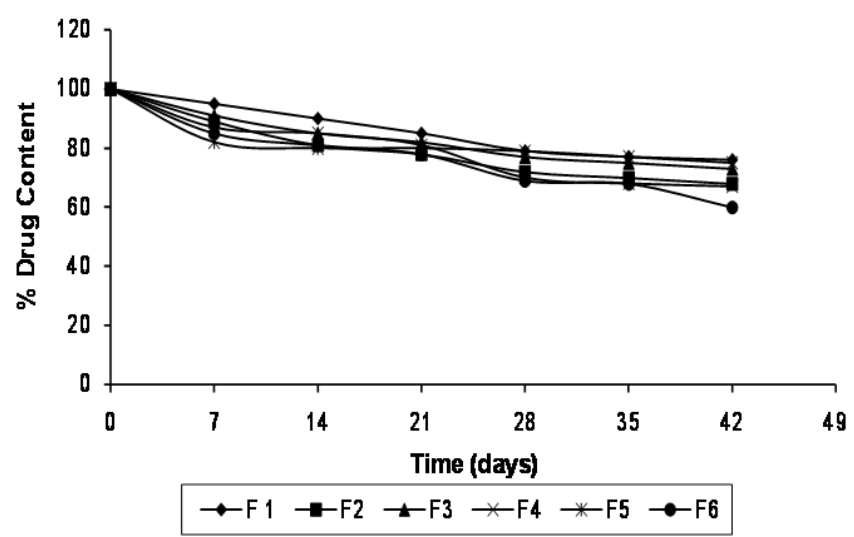

Fig. 4 : Stability Studies of Different Formulations at Room temperature. order of mucoadhesion for the formulations were as found to be $\mathrm{F} 1>\mathrm{F}_{2}>\mathrm{F}_{4}>\mathrm{F}_{5}>\mathrm{F}_{6}>\mathrm{F}_{3}$. The mucoadhesion profile of the formulations is depicted in for the study period of $3.5 \mathrm{~h}$

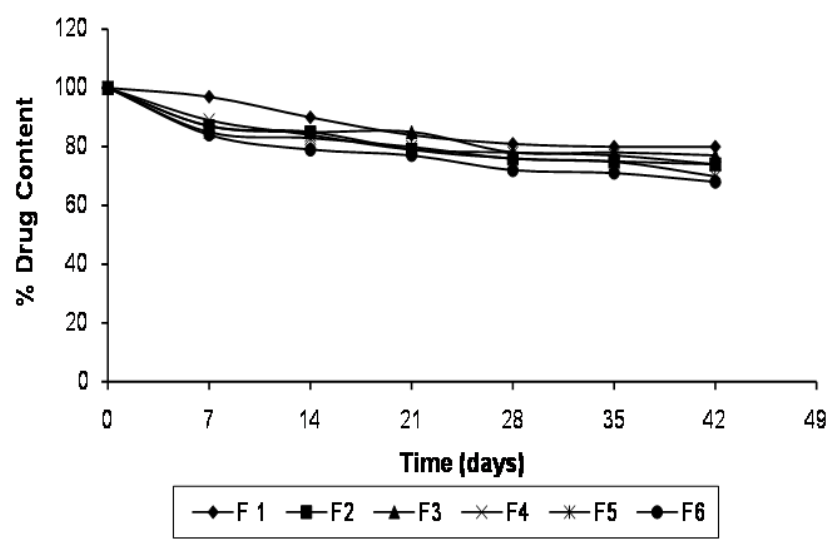

Fig. 5 : Stability Studies of Different Formulations at Refrigerated Temperature 


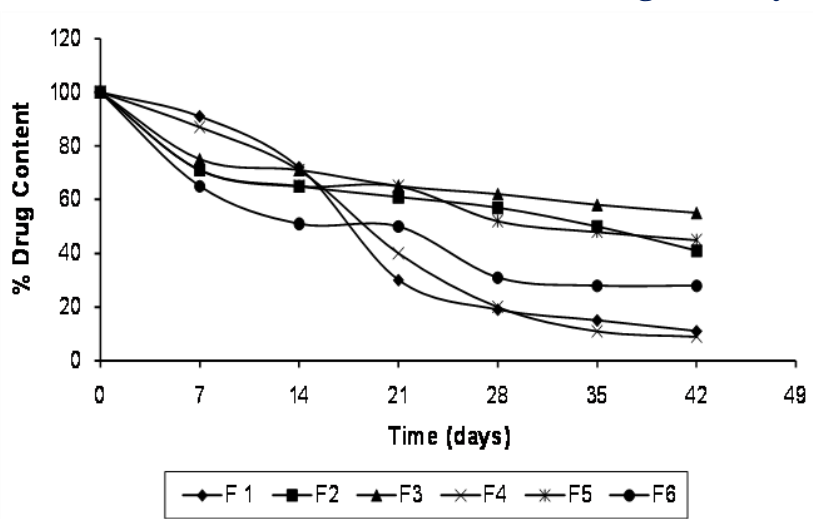

Fig. 6 : Stability Studies of Different Formulations at 45 C

The stability studies of formulations were performed at three different temperature (refrigerated temperature, room temperature and $45{ }^{\circ} \mathrm{C}$ ) for six week after 7,14 , $21,28,35$ and 42 day of storage the drugs content in the microspheres were determined. Fig.4,5 and 6

It was found that at refrigerated temperature microspheres are stable as compared that in room temperature and $45{ }^{\circ} \mathrm{C}$. Microspheres showed maximum loss of drug content at $45{ }^{\circ} \mathrm{C}$ due to the enhanced rate of degradation of Levofloxacin at elevated temperature. It was concluded that incorporation of drug into the polymeric matrix of microspheres improved the stability of the drug. Formulation $F_{1}$ showed the best stability profile at refrigerated and at room temperature while at elevated temperature decrease in the stability was observed. It was found that the formulation $F_{2}, F_{3}$ and $F_{5}$

\section{REFERNCES}

1. Vyas SP, Khar RK, Targeted and Controlled Drug Delivery. $1^{\text {st }}$ ed. New Delhi:, CBS Publishers Distributors; 2002.P. 417-54.

2. Das NG, Das SK, Controlled Release of Oral Dosage Forms, Pharm. Tech. $6^{\text {st }}$ ed. New Delhi; CBS Publishers Distributors; 2003.P.10-16.

3. Venkateswariu V, Reddy PR, Lipid Microspheres as Drug Delivery System: A Review, IJPS, 2001,63(6), 450-58.

4. Kamath KR, Park K, In Swarbrick J, Boylan JC, Eds., Encyclopedia of pharmaceutical technology. Vol. 10. Newyork: Marcel Dekker, 1990.P.133-63 .

5. Gandhi RB, Robinson JR, Bioadhesion in Drug Delivery: A Review, IJPS, 1998 50( 3), 145-52. showed the good stability profile as compared to other formulations at the same conditions.

Among the mucoadhesive microspheres of Levofloxacin hydrochloride prepared using SCMC, sodium Alginate and Carbopol-940 polymers, the formulations $F_{1}$ and $F_{2}$ containing SCMC and CP-940 showed reproducible results and the best mucoadhesive profile with good surface morphology. Formulation $\mathrm{F}_{2}$ (Carbopol-940), $\mathrm{F}_{3}$ (Sodium alginate) and $\mathrm{F}_{5}$ (SCMC / Sodium alginate) microspheres showed the optimum, sustained release pattern as well as the good stability profile.

\section{ACKNOWLEDGEMENTS}

This work was supported by the School of Pharmacy, B.I.T,Partapur-Meerut(UttarPradesh), India.

\section{CONCLUSION}

Mucoadhesive microspheres of Levofloxacin hydrochloride were designed using different polymers. Microspheres showed maximum loss of drug content at $45{ }^{0} \mathrm{C}$ due to the enhanced rate of degradation of Levofloxacin at elevated temperature. It was concluded that incorporation of drug into the polymeric matrix of microspheres improved the stability of the drug. Formulation $F_{1}$ showed the best stability profile at refrigerated and at room temperature while at elevated temperature decrease in the stability was observed. It was found that the formulation $F_{2}, F_{3}$ and $F_{5}$ showed the good stability profile as compared to other formulations at the same conditions. Further studies can be performed as invivo drug release in animals in future.
6. Khanna R Agarwal, SP Ahuja, Mucoadhesive Buccal drug delivery: A Potential Alternative to Conventional Therapy, IJPS, 1998, 60(1), 1-11.

7. Burger DJ, Hickey AJ, In Swarbrick J, Boylan JC, Eds., Encyclopedia of Pharmaceutical Technology, Vol. 10, 1990, Marcel Dekker, Newyork,1-29.

8. Gandhi RB, Robinson JR, Bioadhesion in Drug Delivery, IJPS, 1988, 50(1), 147-148.

9. Khar RK, Ahuja A, Ali J In, Jain NK , Eds , Controlled and Novel Drug Delivery. New Delhi: CBS Publishers \& Distributors, 2002.P. 353-355. 